loro, ma, soprattutto, che siano condotte in modo tale da evitare come spesso è successo in passato con gli Stati Uniti e, in parte, il Giappone - l'esclusione di «casi speciali», qualunque sia la ragione per qualificarli come tali. A quel punto, la questione più interessante sarà se $i$ contributi teorici che Ware ha passato in rassegna in questo suo libro dimostreranno di «tenere» anche al di là delle liberal-democrazie del mondo occidentale e industrializzato dove sono stati fin qui applicati.

\title{
[Alessandro Chiaramonte]
}

FRANCESCA ZAJCZYK, Fonti per le statistiche sociali, Milano, Angeli, 1996, pp. 217.

Il volume di Francesca Zajczyk è il settimo della collana Metodologia delle Scienze Umane, diretta da Alberto Marradi.

Scopo del volume è offrire una rassegna delle principali fonti statistiche nazionali e internazionali (copertura, altre caratteristiche, enti produttori, norme vigenti, ecc.), mettendone in rilievo pregi e limiti di natura sia metodologica sia sostanziale. Il testo è corredato da appendici e rappresentazioni grafiche utili e interessanti per il ricercatore sociale (si veda per esempio la figura 5 sul rapporto tra abitanti del capoluogo e del resto della provincia in alcune città).

Il primo capitolo ricostruisce lo sviluppo storico della statistica come attività cognitiva e illustra le principali caratteristiche dell'analisi secondaria; quest'ultima consiste (come aveva già sottolineato Hyman, qui ripreso dall'A.) «in un'estrazione di conoscenza su argomenti diversi da quelli che costituiscono il focus dei sondaggi originari» (p. 13).

Nel secondo capitolo viene ricostruita l'evoluzione dell'informazione statistica in Italia, in particolare la Zajczyk si sofferma sulla natura e i compiti dell'Istat, ponendo in rilievo lo spirito e gli effetti della riforma del 1989 che ha introdotto nel nostro Paese il Sistan (Sistema Statistico Nazionale). Nelle intenzioni del legislatore il Sistan deve facilitare la costruzione di un più ampio archivio che comprenda tutta quella massa di dati che, prodotti in maniera disomogenea da amministrazioni pubbliche ed enti di varia natura, finiscono per non essere cumulabili e confrontabili, e pertanto poco utili a fini analitici.

Il terzo capitolo si sofferma sulle forme standard di raccolta di dati ufficiali: anagrafi, censimenti, archivi amministrativi. Degli archivi amministrativi l'A. denuncia «l'eccessiva rigidità con cui sono organizzati; con la conseguente difficoltà di collegare i dati di files diversi, e il condizionamento da parte di norme legislative cui la registrazione delle informazioni è vincolata, con il conseguente problema del rispetto della privacy» (p. 50).

Il collegamento tra files di origine amministrativa e files provenienti da indagini di natura conoscitiva in Italia è pressoché inesisten- 
te, e le ripercussioni negative di questo fatto sono pesanti. Giustamente l'A. denuncia come nel nostro Paese permanga ancora molto forte «il senso di possesso del dato» e sia assente la consapevolezza dell'importanza del «dato statistico come risorsa anziché come bene».

Il quarto capitolo affronta il delicato argomento delle unità territoriali di analisi e dei molteplici e disparati criteri con cui è suddiviso a livello amministrativo il territorio nazionale; come osserva l'A. «un mosaico di livelli e di suddivisioni in zone». Nel quinto capitolo sono passati in rassegna una serie di aspetti inerenti ai dati che l'autrice riconduce al concetto di qualità dei dati, introdotto in metodologia dallo statunitense Naroll (Data Quality Controll, 1962). Giustamente è sottolineata la complessa intenzione del concetto (adeguatezza, validità, sensibilità, fedeltà, comparabilità): «la qualità è un concetto multidimensionale, sia perché si esprime in numerose caratteristiche relative all'oggetto al quale viene riferito, sia perché risulta largamente correlato alle finalità per le quali l'oggetto è usato» (p. 90).

Il sesto capitolo individua per ciascuna area tematica le indagini prodotte dai vari soggetti, con i relativi pregi e limiti. Insieme agli elenchi nelle appendici, contenenti percorsi e caratteristiche dei modelli di rilevazione e livello territoriale di riferimento, il capitolo si segnala per il contributo che fornisce alla conoscenza delle fonti ufficiali in merito alle diverse aree di indagine.

Il settimo capitolo analizza il tema dei giovani con particolare riferimento alle fonti di informazioni su di essi presenti a livello locale.

Il volume fornisce anche gli indirizzi dei principali enti produttori di statistiche sociali, le definizioni impiegate negli ultimi quattro censimenti per rilevare alcuni fenomeni e un glossario (curato insieme con Marradi) dei principali termini metodologici.

In conclusione Fonti per le statistiche sociali si segnala come un prezioso strumento di lavoro, anche per l'accuratezza con cui sono evidenziati i limiti metodologici e sostanziali delle statistiche sociali prodotte nel nostro Paese: come esempio dei primi basti ricordare la disomogeneità delle unità territoriali di rilevazione, che influisce sulla comparabilità sincronica e diacronica. Quanto ai limiti sostanziali l'A. rileva la presenza di «interi settori per i quali non sembrano esistere informazioni a livello comunale e, in alcuni casi, neppure provinciale: ciò avviene, per esempio, per l'assistenza, la sanità e la cultura» (p. 106); anche settori di moda come la qualità della vita non sembrano essere indagati a sufficienza. 